allein besorgte, es verstanden, die neuesten Forschungsergebnisse in durchaus leichtfaßlicher Weise und dabei in gedrängter Kürze heranzuziehen, insbesondere clie großen Errungenschaften der letzten Zeit auf dem Gebiete der Erkenntnis vom Aufbau der Materie und der Relativitätstheorie in mustergïltiger Weise vorzubringen. Der vorliegende zweite Band enthält die Abschnitte über Magnetismus, die ruhende Elektrizität (Elektrostatik), die strömende Elektrizität (Elektrodynamik), Umwandlung. elektrischer Energie in Wärmeenergie durch den Strom, Elektrolyse, Elektromagnetismus, mechanische Wirkungen des elektrischen Stromes, Induktion, Elektrische Ladungen, die atmosphärische Elektrizität, elelstrische Schwingungen, Einführung in die Relativitätstheorie sowie die Anschauungen und Hilfsvorstellungen von dem. Wesen der Elektrizität im Wandel der Zeiten, woran sich als Anhang Tabellen über wichtige physikalische Konstanten und Zahlentabellen schließen. Letztere Tabellen sind gewiß willkommen, aber naturgemäß mit einer gewissen Willkür zusammengestellt und könnten vielleicht im Hinblick auf die analogen in dem bekannter allgemein verbreiteten Lehrbuch E. Kohlrausch und den Landolt-Börnsteinschen etc. zugunsten von Texterweiterungen in zulzünftigen Auflagen zurücktreten. Nur nebenbei sei angeführt, daß S. 577 Franz Exner mit Felix M. Exner verwechselt wurde. Jedenfalls ist das gesammte Werk eines der vortrefflichsten der in- und ausländischen einführenden physikalischen Literatur.

St. Meyer.

\title{
Lehrbuch der Physik zum Gebrauche beim Unterricht, bei akademischen Vorlesungen und zum Selbststudium. Von
} E. Grimsehl in zwei Bänden. Erster Band: Mechanik, Wärmelehre, Akustik und Optik. Sechste, vermehrte und verbesserte Auflage. Herausgegeben von W. Hillers unter Mitarbeit von H. Starke. Mit 1090 Fig. im Text und 10 Fig. auf zwei farbigen Tafeln. XII + 1142 S. Verlag von B. G. Teubner, Leipzig 1923.

Die rasch aufeinanderfolgenden Auflagen sprechen am besten fü die Beliebtheit dieses Werkes. - Als Erweiterungen gegenüber der III. Auflage (besprochen in den Literaturberichten dieser Zeitschrift, Jahrgang XXVIII, S. 10, 1917) wird im Vorwort folgendes hervorgehoben: $\gg$ Die einzige tiefgreifende Anderung besteht in der neuen Darstellung der neuzeitlichen Anschauungen über den Bewegungswiderstand in Flüssigkeiten und Luft, welche in den Händen von Kutta, Joukowski und L. PrandtI endlich zu einer mit der Erfahrung gut übereinstimmenden Tragflächentheorie gefühtt haben. Das Bestreben, diese Anschauungen in geschlossenem Aufbau darzubieten, machte die Einführung und die Entwicklung zahlreicher neuer Begriffe notwendig, so daß dem Gegenstande acht neue Paragraphen gewidmet werden mußten. Im allgemeinen wurde bei der Neubearbeitung niemals die Richtschnur aus dem Auge verloren, einer Unfangsvermehrung des Bandes zu steuern. Die neuen experimentellen Ergebnisse, besonders der Göttinger Schule, über die Widerstands- und Auftriebsmessungen schienen uns aber in allgemein physikalischer Hinsicht so bedeutungsvoll, die notwendigen Auseinandersetzungen über Potentialströmung, Drehströmung, Stromlinien, Stromfäden und Drucke sowie die eigentliche Auftriebstheorie schienen uns in Hinblick auf die ganz entsprechenden Begriffe und Lehren des elektromagnetischen Feldes so lehrreich, daß wir demgegenüber glaubten, die unerwïnschte Umfangsvermehrung mit in den Kauf nehmen zu müssen. «

Falls der Referent bezüglich einer nächsten Auflage eine Anregung geben darf, möchte er vorschlagen, die nicht ganz gegliickten theoretischen Erörterungen ïber das Plancksche Strahlungsgesetz (S. 1077-1088) zu streichen und dafür die fundamental wichtigen empirischen Gesetze über die Serienspektra und ihr Verhalten im elektrischen und magnetischen Feld aufzunehmen.

H. Thirring.

Lehrbuch der praktischen Physik. Von Friedrich Kohlrausch. Vierzehnte, stark vermehrte Auflage ( $47-51$ Tausend), neu bearbeitet von E. Brodhun, H. Geiger, E. Giebe, E. Grüneisen, L. Holborn, K. Scheel, O. Schönrock und E. Warburg. Mit 395 Figuren im Text, XXVIII + 802 S. Verlag von B. G. Teubner, Leipzig-Berlin. 